\title{
Damping of metallized bilayer nanomechanical resonators at room temperature
}

\author{
Maximilian J. Seitner, Katrin Gajo, and Eva M. Weig ${ }^{\text {a) }}$ \\ University of Konstanz, Department of Physics, 78457 Konstanz, Germany
}

(Received 19 September 2014; accepted 12 November 2014; published online 24 November 2014)

\begin{abstract}
We investigate the influence of gold thin-films subsequently deposited on a set of initially bare, doubly clamped, high-stress silicon nitride string resonators at room temperature. Analytical expressions for resonance frequency, quality factor and damping for both in- and out-of-plane flexural modes of the bilayer system are presented, which allows for the determination of effective elastic parameters of the composite structure from our experimental data. We find the inverse quality factor to scale linearly with the gold film thickness, indicating that the overall damping is governed by losses in the metal. Correspondingly, the mechanical linewidth increases by more than one order of magnitude compared to the bare silicon nitride string resonator. Furthermore, we extract mechanical quality factors of the gold film for both flexural modes and show that they can be enhanced by complete deposition of the metal in a single step, suggesting that surface and interface losses play a vital role in metal thin-films. (C) 2014 AIP Publishing LLC.
\end{abstract}

[http://dx.doi.org/10.1063/1.4902430]

Nanoelectromechanical systems (NEMS) enable both the study of fundamental physical effects and future applications as integrated devices, e.g., in the field of ultra sensitive sensor technology. ${ }^{1}$ As an important representative of such structures, freely suspended nanomechanical string resonators are exploited for their remarkable mechanical properties, providing high quality factors even at room temperature. ${ }^{2,3}$ The overall performance of nanomechanical resonators is governed by their mechanical damping, originating from the combination of several fundamentally different loss mechanisms. ${ }^{4}$ In general, extrinsic and intrinsic losses are distinguished, both of which have been studied extensively during the last decades. Intrinsic losses, such as thermoelasticity, ${ }^{5}$ clamping loss, ${ }^{6,7}$ surface, ${ }^{8,9}$ and volume defects such as twolevel system (TLS) damping ${ }^{10-12}$ can be partially mitigated by intelligent resonator design. Extrinsic loss sources, like transduction mediated damping ${ }^{13,14}$ can be reduced, e.g., through elimination of metallization layers on the resonant structure or the use of different detection techniques, such as optical schemes. However, hybrid nanostructures frequently rely on such metallization films, providing functionalization for coupling mechanical resonators to other degrees of freedom. ${ }^{15-17}$ For those purposes, it is inevitable to acquire a deeper understanding of the metal's impact on the overall damping of the system ${ }^{18-21}$ as well as its influence on elastic parameters, ${ }^{22}$ especially at room temperature.

Here, we present a thorough investigation of metallized bilayer nano-resonators based on doubly clamped, highstress silicon nitride string resonators at room temperature. Starting from the basic assumptions of Euler-Bernoulli beam theory, ${ }^{23}$ we develop analytical expressions for the in- and out-of-plane harmonic eigenmodes of pre-stressed bilayer systems, which allows us to quantify their elastic constants. Furthermore, a model for the metal-induced quality factor is adapted to our system. Comparing the theoretical findings to

a)eva.weig@uni-konstanz.de our experimental data, the vast influence of the metal coating on the bare, non-metallized resonant structure becomes apparent. In the future, the resulting quantitative understanding will allow for the engineering of resonators with pre-defined frequency and quality factor by choosing the most suitable bilayer composition and resonator dimensions.

A typical device is depicted in Fig. 1. We fabricate doubly clamped, freely suspended silicon nitride strings of different lengths on a silicon substrate in a top-down approach, using standard electron beam lithography and different dry and wet etching techniques. The LPCVD-grown amorphous silicon nitride on a sacrificial layer atop the silicon substrate is intrinsically pre-stressed; hence, our resonators have a high inherent tensile stress of $830 \mathrm{MPa}^{3}$ The bare silicon nitride strings have a thickness of $d_{1}=100 \mathrm{~nm}$ and a width of $b=250 \mathrm{~nm}$, while their length varies from $L_{0}=33 \mu \mathrm{m}$ to $L_{0}=53 \mu \mathrm{m}$. The resonances of their flexural modes are measured in an optical interferometer setup, where we refer to the oscillation in z-direction as out-of-plane (oop) and in

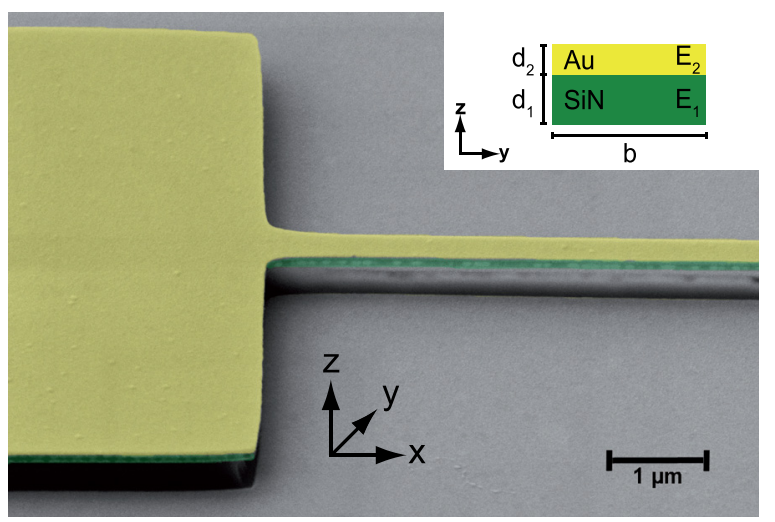

FIG. 1. False color SEM micrograph of part of a doubly clamped silicon nitride string resonator covered with $50 \mathrm{~nm}$ gold. Picture is taken under a tilted angle to point out the bilayer structure and the free suspension. Inset shows a schematic cross section of the resonator, introducing the geometric parameters width $b=250 \mathrm{~nm}, \mathrm{SiN}$ thickness $d_{1}=100 \mathrm{~nm}$ and Au thickness $d_{2} . E_{1}$ and $E_{2}$ represent the respective Young's moduli. 
y-direction as in-plane (ip) mode. After this first characterization, we successively deposit gold on the sample by electron beam evaporation to sequentially increase the total thickness $d_{2}$ of the metal. A $3 \mathrm{~nm}$ adhesion layer of chromium between the $\mathrm{SiN}$ resonator and the first gold layer is neglected in the following analysis. ${ }^{4}$ For each gold film thickness, we subsequently record the resonances of the now bilayer system and extract the characteristic oscillation parameters from Lorentzian fits, again using optical interferometric detection. Consequently, we end up with results for nine different gold layer thicknesses on the same sample, as well as the data for the non-metallized SiN strings. Those results are evaluated according to a theoretical model of doubly clamped, prestressed bilayer nanomechanical resonators (see supplementary material ${ }^{24}$ for detailed derivation). Starting from the basic assumptions of Euler-Bernoulli beam theory, ${ }^{23,25,26}$ we can express the bilayer system's resonant frequencies for both inand out-of-plane flexural mode polarizations as ${ }^{24}$

$$
f_{0, j}=\frac{j^{2} \pi}{2 L^{2}} \sqrt{\frac{(E I)_{\mathrm{eff}}}{(\rho A)_{\mathrm{eff}}}} \sqrt{1+\frac{(\sigma A)_{\mathrm{eff}} L^{2}}{j^{2}(E I)_{\mathrm{eff}} \pi^{2}}},
$$

with the harmonic mode index $j=1,2,3, \ldots$ and effective material and elastic parameters $(\rho A)_{\text {eff }},(\sigma A)_{\text {eff }}$ and $(E I)_{\text {eff. }}$ The effective density is

$$
(\rho A)_{\mathrm{eff}}=\frac{\rho_{1} d_{1}+\rho_{2} d_{2}}{d_{1}+d_{2}} A=\tilde{\rho} A,
$$

and the effective tensile stress reads

$$
(\sigma A)_{\mathrm{eff}}=\frac{\sigma_{1} d_{1}+\sigma_{2} d_{2}}{d_{1}+d_{2}} A=\tilde{\sigma} A,
$$

where $A=b\left(d_{1}+d_{2}\right)$ equals the cross section area of the bilayer (see inset of Fig. 1). The effective bending rigidity depends on the polarization of the flexural mode, ${ }^{24}$ namely,

$$
\begin{aligned}
& (E I)_{\text {eff }, \text { oop }} \\
& \quad=b \frac{E_{1}^{2} d_{1}^{4}+2 E_{1} E_{2} d_{2}\left(2 d_{1}^{3}+2 d_{1} d_{2}^{2}+3 d_{1}^{2} d_{2}\right)+E_{2}^{2} d_{2}^{4}}{12\left(E_{1} d_{1}+E_{2} d_{2}\right)} \\
& =E_{1} I_{\text {eff }}^{\text {oop }},
\end{aligned}
$$

for out-of-plane, and

$$
(E I)_{\mathrm{eff}, \mathrm{ip}}=\frac{b^{3}\left(E_{1} d_{1}+E_{2} d_{2}\right)}{12}=E_{1} I_{\mathrm{eff}}^{\mathrm{ip}},
$$

for in-plane oscillations. For $\sigma_{1}=\sigma_{2}=0$, we recover the same result as reported for stress-free bilayers, ${ }^{4,27}$ whereas for high tensile stress and low harmonic modes of high aspect ratio beams

$$
\frac{(\sigma A)_{\mathrm{eff}} L^{2}}{j^{2}(E I)_{\mathrm{eff}} \pi^{2}} \gg 1
$$

such that Eq. (1) reduces to

$$
f_{0, j} \approx \frac{j}{2 L} \sqrt{\frac{\tilde{\sigma}}{\tilde{\rho}}}=\frac{j}{2 L} \sqrt{\frac{\sigma_{1} d_{1}+\sigma_{2} d_{2}}{\rho_{1} d_{1}+\rho_{2} d_{2}}},
$$

which is similar to the findings in Ref. 28 assuming only a tensile stress of the SiN layer. Using the established values for the material constants of our silicon nitride strings, ${ }^{3}$ density $\rho_{1}=2.8 \mathrm{~g} / \mathrm{cm}^{3}$, Young's modulus $E_{1}=160 \mathrm{GPa}$, tensile stress $\sigma_{1}=830 \mathrm{MPa}$, and literature values for the gold films, ${ }^{29} \rho_{2}=19.3 \mathrm{~g} / \mathrm{cm}^{3}$ and $E_{2}=78 \mathrm{GPa}$, we are now able to fit the theoretical predictions to our experimental data. We used two fit parameters, the tensile stress $\sigma_{2}$ of the gold film, as well as the length of the resonator $L$. Note that $L$ does not coincide with the nominal length of the resonator $L_{0}$ as a result of the undercut of the clamping points during the HF wet etch $^{7}$ (cf. Fig. 1). Exemplary results are depicted in Fig. 2 showing the fundamental out-of-plane eigenfrequencies of a nominally $L_{0}=53 \mu \mathrm{m}$ and of a $L_{0}=35 \mu \mathrm{m}$ long resonator as well as the corresponding in-plane flexural mode of the $53 \mu \mathrm{m}$ string as a function of gold film thickness. We find the data in good agreement with the theoretical predictions. The obtained values of $L$ consistently exceed $L_{0}$ with the exception of one data point, which is considered as outlier. ${ }^{24}$ The effective elongation $\left(L-L_{0}\right)$ is more pronounced for shorter strings and for out-of-plane modes, ${ }^{24}$ reflecting the larger impact of the underetched clamping region on vibrations perpendicular to the sample plane. The inset of Fig. 2 displays the tensile stress in the gold film for resonators of different lengths extracted from the fit. We find positive values for $\sigma_{2}$ in the out-of-plane, and slightly negative values for the in-plane mode, both with a tendency towards smaller $\sigma_{2}$ for longer resonators. The overall magnitude of $\sigma_{2}$ lies well within the range of stress expected for e-beam evaporated gold films. ${ }^{30}$ This stress originates from the evaporation of the gold and should not depend on resonator length. The dependence of $\sigma_{2}$ on length and mode polarization remains topic of further investigation. However, since the extracted stress in the gold film is very small compared to the residual stress in the silicon nitride $(<4 \%)$, this effect may well be interpreted as an artifact in our fitting routine of Eq. (1) to Eq. (5).

Metallizing nanomechanical resonators not only affects their resonant frequencies. The metal's deleterious impact is

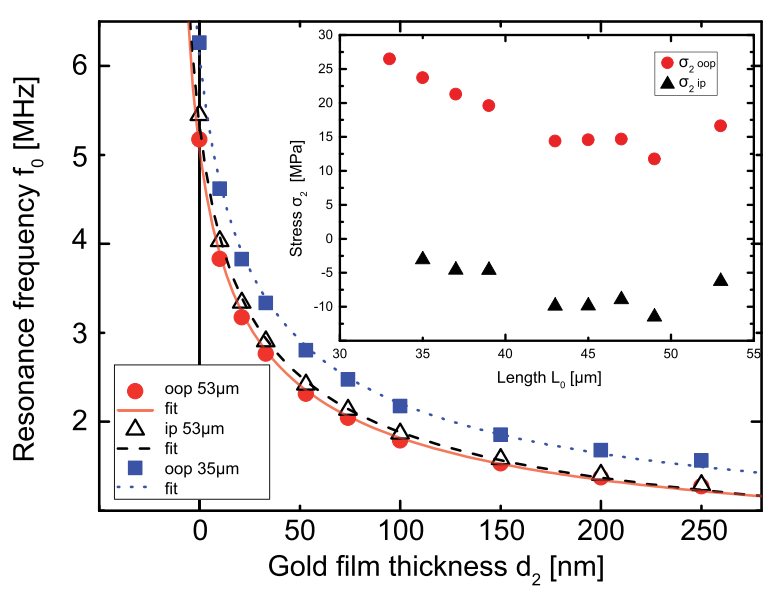

FIG. 2. Decrease of resonance frequency as a function of gold film thickness, for the fundamental out-of-plane flexural modes of a $53 \mu \mathrm{m}$ (red dots) and a $35 \mu \mathrm{m}$ (blue squares) as well as the $53 \mu \mathrm{m}$ long resonator's corresponding fundamental in-plane mode (open black triangles). Red solid, blue dotted, and black dashed lines represent the fit of Eq. (1) to the data, respectively. The inset shows fitted tensile stress of gold films versus resonator length for in-plane (black triangles) and out-of-plane mode (red dots). 
most pronounced in the otherwise high room temperature mechanical quality factor (Q-factor) of our silicon nitride string resonators. The past decades showed extensive studies on metal thin-film damping, mostly at low temperatures. ${ }^{4,18-21,31-33}$ According to this framework, we describe the metal's impact on the inverse quality factor $1 / \mathrm{Q}$. Here, one should point out deliberately that by $1 / \mathrm{Q}$, we refer to the inverse quality factor and not the damping. We find $1 / \mathrm{Q}$ of a bilayer structure to equal ${ }^{4,31,34}$

$$
\frac{1}{Q}=\frac{1}{1+\beta}\left(\frac{1}{Q_{0}}+\beta \frac{1}{Q_{m}}\right),
$$

where $Q_{0}$ represents the Q-factor of the non-metallized resonator and $Q_{m}$ an effective mechanical Q-factor of the metal film comprising all relevant loss mechanisms in the metal. The dimensionless factor $\beta=\left(E_{2} d_{2}\right) /\left(E_{1} d_{1}\right)$ depends on the ratio of the material's Young's moduli and thicknesses and can be interpreted as the ratio of the effective bilayer thicknesses within the framework of transformed sections. ${ }^{25}$ Since in our case $\beta$ is typically small, we can approximate Eq. (8) in a Taylor series expansion to first order in $\beta$,

$$
\frac{1}{Q}=\frac{1}{Q_{0}}+\frac{E_{2}}{E_{1} Q_{0}}\left(\frac{Q_{0}}{Q_{m}}-1\right) \frac{d_{2}}{d_{1}} .
$$

This equation clearly shows that the inverse quality factor of the bilayer system scales linearly with the gold film thickness $d_{2}$ in the limit of thin metal films, i.e., small $\beta$. This behavior is experimentally validated in Fig. 3. Here, we plot the inverse mechanical Q-factor as a function of the gold film thickness $d_{2}$. Since Eq. (9) holds only for small values of $\beta$, we consider only the first five data points for fitting (red solid line), such that $d_{2} \leq 50 \mathrm{~nm}$, which yields $\beta<0.25$. For thicker films, our approximation breaks down and $1 / Q$ begins to deviate from the first order expansion. Note that this effect gets more pronounced for higher harmonic modes. ${ }^{24}$ From the fit of Eq. (9) (red solid line), we extract values of $Q_{m}$, which are interpreted as effective Q-factors of the metal. ${ }^{31-33,35}$ As displayed in Figs. 3(b) and 3(c), $Q_{m}$ tends to increase approximately linear with increasing resonator length and therefore decreasing resonant frequency. This behavior is in qualitative agreement with that of the bare SiN strings (red triangles in Figs. 3(b) and 3(c)) and is already known from defect damping dominated systems, ${ }^{2,36,37}$ indicating that the bilayer system's damping is governed by defects in the metal. The contribution from other fundamental loss mechanisms, such as thermoelasticity, ${ }^{5}$ is significantly smaller than the measured $Q_{m}{ }^{38}$ Overall, the observed effective room temperature mechanical quality factors of the gold films of several thousands agree well with literature findings from different metallic systems. $^{31-33,35}$

In order to investigate the metal induced $Q_{m}$ in a more detailed way, we examine two reference samples where gold films of $51 \mathrm{~nm}$ and $100 \mathrm{~nm}$ thickness (green stars and black diamonds in Fig. 3(a)) have been evaporated on the sample in a single step, respectively. These structures yield metal quality factors up to $Q_{m}=20000$ for out-of-plane and $Q_{m}=4000$ for in-plane flexural fundamental modes. Compared to the case of subsequently evaporated gold films, single step evaporation results in a significant improvement of the Q-factor of the out-of-plane oscillation direction, whereas the Q-factor of the in-plane oscillation remains rather unaffected. We attribute this to the impact of surface losses. ${ }^{9}$ Subsequent evaporation of several gold layers creates additional interfaces. Furthermore, the sequential investigation implies exposing the device to air, which leads to additional contamination layers along the out-of-plane oscillation direction, yielding more damping and therefore, lower Q-factors for out-of-plane vibrations. Contrary, the additional interfaces comprehensively have less impact on inplane vibrations.

Since the metal's quality factors $Q_{m}$ are significantly smaller than the bare silicon nitride $Q_{0}$ 's, one can approximate the second term of Eq. (9) even further

$$
\frac{E_{2}}{E_{1} Q_{0}}\left(\frac{Q_{0}}{Q_{m}}-1\right) \frac{d_{2}}{d_{1}} \approx \frac{1}{Q_{m}} \frac{E_{2}}{E_{1}} \frac{d_{2}}{d_{1}} .
$$

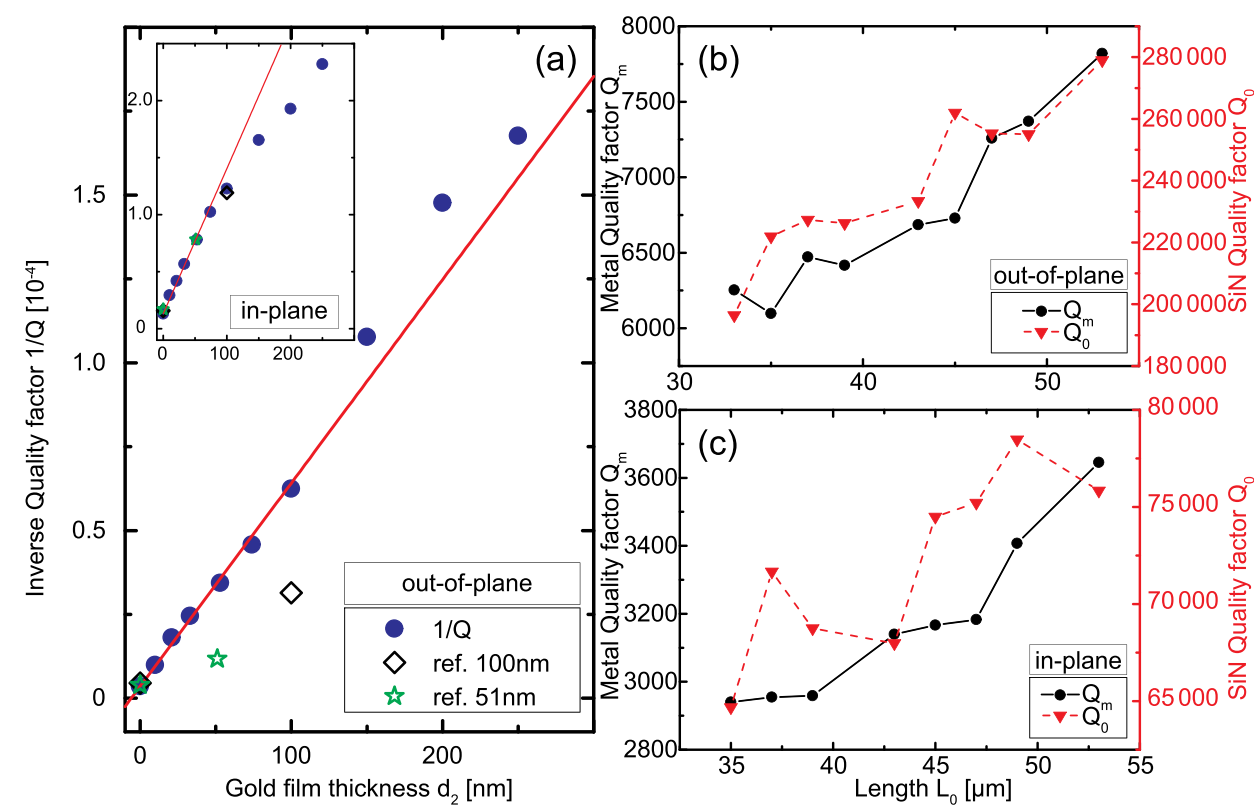

FIG. 3. (a) Inverse Q-factor versus gold film thickness, including reference samples with $51 \mathrm{~nm}$ (green stars) and $100 \mathrm{~nm}$ (black diamonds) thick gold films for out-of-plane fundamental flexural mode of a $53 \mu \mathrm{m}$ long resonator. Red solid line depicts fit to the 5 first data points with $\beta<0.25$. Inset shows corresponding in-plane mode. (b) and (c) Metal Q-factor (black circles, left scale) vs. resonator length. Red triangles (right scale) show Qfactors of non-metallized resonators for comparison, respectively. 


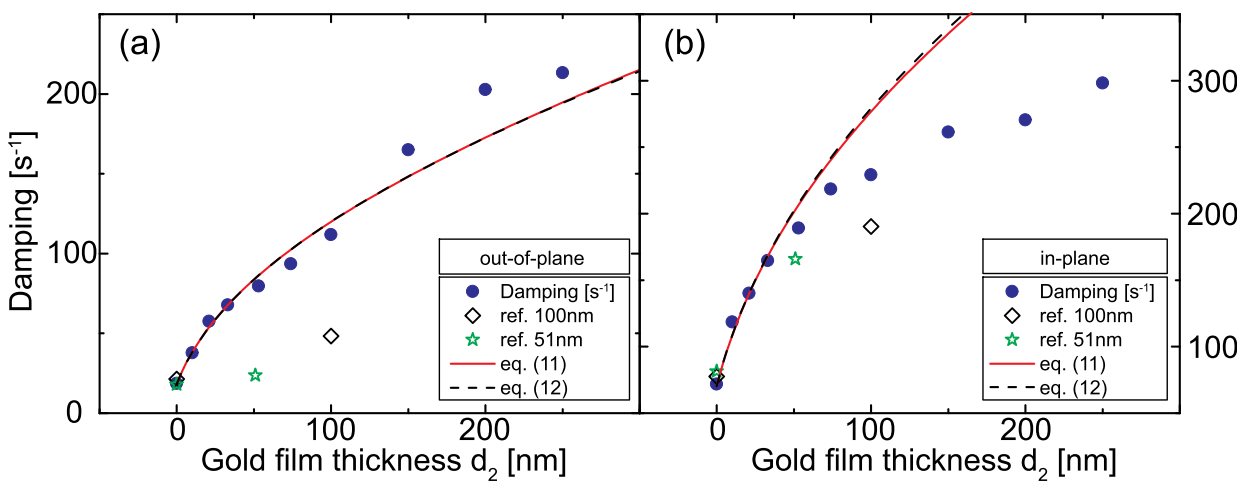

FIG. 4. Damping, i.e., resonance linewidth, versus gold film thickness. (a) Out-of-plane fundamental flexural mode of a $53 \mu \mathrm{m}$ long resonator. (b) Corresponding in-plane mode. Experimental data (blue dots) including reference samples with $51 \mathrm{~nm}$ (green stars) and $100 \mathrm{~nm}$ (black diamonds) thick gold films. Red solid (black dashed) lines have been calculated with no free parameters from Eqs. (11) and (12), respectively.
The damping constant, defined in units of frequency, corresponds to the linewidth of the mechanical resonance and can hence be expressed as

$$
\gamma_{j}=\frac{f_{0, j}}{Q}=f_{0, j}\left(\frac{1}{Q_{0}}+\frac{1}{Q_{m}} \frac{E_{2}}{E_{1}} \frac{d_{2}}{d_{1}}\right) .
$$

Using the experimentally determined and material parameters from above, the bilayer system's damping can now be calculated from Eq. (11) with no free parameters. Fig. 4 displays the resulting damping curve (red line), along with the experimental data. We find excellent agreement between experiment and theoretical assumption for the first five data points of flexural modes where the first order approximation of $\beta$ still holds. For the case of the strongly stressed string, Eq. (11) can be approximated using Eq. (6), yielding

$$
\gamma_{j}=\frac{j}{2 L} \sqrt{\frac{\sigma_{1} d_{1}+\sigma_{2} d_{2}}{\rho_{1} d_{1}+\rho_{2} d_{2}}}\left(\frac{1}{Q_{0}}+\frac{1}{Q_{m}} \frac{E_{2}}{E_{1}} \frac{d_{2}}{d_{1}}\right) .
$$

The dashed line in Fig. 4 has been obtained from the highstress approximation in Eq. (12). It coincides with the red curve, confirming that the elastic properties of the metallized bilayer system are indeed dominated by the high effective tensile stress in the material. Taking into account that the total damping amounts to the sum of all contributing damping mechanisms, ${ }^{4}$ Eqs (11) and (12) can be interpreted as the sum of the damping in $\mathrm{SiN}$ and gold, respectively. Indeed, the first term in Eq. (12) accounts for the damping of the non-metallized $\mathrm{SiN}$ string resonator. The second term is governed by the quality factor assigned to the metal film as well as the ratio of the effective thicknesses. ${ }^{25}$

In conclusion, we have investigated the mechanical properties of gold-coated silicon nitride string resonators at room temperature. Using analytical expressions for resonant frequency, quality factor, and damping of bilayer structures, we have been able to show that the damping of metallized bilayer nanomechanical string resonators is governed by defect losses in the metal film and scales with its thickness. Comparison of the subsequently evaporated gold coatings with reference samples evaporated in a single step revealed that surface losses play an important role in metal dissipation. The presented thorough analysis enables engineering metallized bilayer resonators with custom eigenfrequency and quality factor by adjusting the resonator dimensions and metal thickness. This is of utmost importance for future applications of metallized resonators in hybrid nanomechanical systems, where a good understanding of the mechanical system is required, for example the functionalization for biosensing applications ${ }^{39}$ or the electromagnetic coupling of NEMS to cold atoms. ${ }^{16,40}$ Furthermore, the above can easily be extended to other metals, enabling to further reduce the metal's damping contribution ${ }^{41}$ or to open up routes towards coupling to other degrees of freedom for hybrid nanosystems by using superconducting ${ }^{15}$ or magnetic ${ }^{17}$ metallizations.

Financial support by the Deutsche Forschungsgemeinschaft via the collaborative research center SFB 767 and the Center of Applied Photonics (CAP) is gratefully acknowledged. We thank James A. Dorman for critically reading the manuscript.

${ }^{1}$ K. L. Ekinci and M. L. Roukes, Rev. Sci. Instrum. 76, 061101 (2005).

${ }^{2}$ S. S. Verbridge, J. M. Parpia, R. B. Reichenbach, L. M. Bellan, and H. G. Craighead, J. Appl. Phys. 99, 124304 (2006).

${ }^{3}$ Q. P. Unterreithmeier, T. Faust, and J. P. Kotthaus, Phys. Rev. Lett. 105, 027205 (2010).

${ }^{4}$ M. Imboden and P. Mohanty, Phys. Rep. 534, 89 (2014).

${ }^{5}$ R. Lifshitz and M. L. Roukes, Phys. Rev. B 61, 5600 (2000).

${ }^{6}$ I. Wilson-Rae, Phys. Rev. B 77, 245418 (2008).

${ }^{7}$ J. Rieger, A. Isacsson, M. J. Seitner, J. P. Kotthaus, and E. M. Weig, Nat. Commun. 5, 3345 (2014).

${ }^{8}$ J. Yang, T. Ono, and M. Esashi, Appl. Phys. Lett. 77, 3860 (2000).

${ }^{9}$ L. G. Villanueva and S. Schmid, e-prints arXiv:1405.6115 [cond-mat.meshall] (2014).

${ }^{10}$ R. O. Pohl, X. Liu, and E. Thompson, Rev. Mod. Phys. 74, 991 (2002).

${ }^{11}$ R. Vacher, E. Courtens, and M. Foret, Phys. Rev. B 72, 214205 (2005).

${ }^{12}$ T. Faust, J. Rieger, M. J. Seitner, J. P. Kotthaus, and E. M. Weig, Phys. Rev. B 89, 100102 (2014).

${ }^{13}$ A. N. Cleland and M. L. Roukes, Sens. Actuators, A 72, 256 (1999).

${ }^{14}$ J. Rieger, T. Faust, M. J. Seitner, J. P. Kotthaus, and E. M. Weig, Appl. Phys. Lett. 101, 103110 (2012).

${ }^{15}$ C. A. Regal, J. D. Teufel, and K. W. Lehnert, Nat. Phys. 4, 555 (2008).

${ }^{16}$ O. Kálmán, T. Kiss, J. Fortágh, and P. Domokos, Nano Lett. 12, 435 (2012).

${ }^{17}$ A. Kamra, M. Schreier, H. Huebl, and S. T. B. Goennenwein, Phys. Rev. B 89, 184406 (2014).

${ }^{18}$ R. Sandberg, K. Mølhave, A. Boisen, and W. Svendsen, J. Micromechan. Microeng. 15, 2249 (2005).

${ }^{19}$ F. W. Beil, R. H. Blick, A. Wixforth, W. Wegscheider, D. Schuh, and M. Bichler, EPL 76, 1207 (2006).

${ }^{20}$ G. Sosale, K. Das, L. Fréchette, and S. Vengallatore, J. Micromechan. Microeng. 21, 105010 (2011).

${ }^{21}$ P.-L. Yu, T. P. Purdy, and C. A. Regal, Phys. Rev. Lett. 108, 083603 (2012).

${ }^{22}$ F. Hocke, M. Pernpeintner, X. Zhou, A. Schliesser, T. J. Kippenberg, H. Huebl, and R. Gross, Appl. Phys. Lett. 105, 133102 (2014).

${ }^{23} \mathrm{~W}$. Weaver, S. P. Timoshenko, and D. H. Young, Vibration Problems in Engineering, 5th ed. (John Wiley \& Sons, 1990).

${ }^{24}$ See supplementary material at http://dx.doi.org/10.1063/1.4902430 for theoretical derivations and additional information.

${ }^{25}$ J. M. Gere and B. J. Goodno, Mechanics of Materials (Cenegage Learning, 2013), Chap. 6.3. 
${ }^{26}$ X.-S. Wang and T.-Y. Zhang, Metall. Mater. Trans. A 38, 2273 (2007).

${ }^{27}$ Y. T. Yang, K. L. Ekinci, X. M. H. Huang, L. M. Schiavone, M. L. Roukes, C. A. Zorman, and M. Mehregany, Appl. Phys. Lett. 78, 162 (2001).

${ }^{28}$ T. S. Biswas, A. Suhel, B. D. Hauer, A. Palomino, K. S. D. Beach, and J. P. Davis, Appl. Phys. Lett. 101, 093105 (2012).

${ }^{29}$ Springer Handbook of Condensed Matter and Materials Data, edited by W. Martienssen and H. Warlimont (Springer, Berlin Heidelberg, New York, 2005).

${ }^{30}$ C. A. Neugebauer, J. Appl. Phys. 31, 1096 (1960).

${ }^{31}$ X. Liu, E. Thompson, B. E. White, Jr., and R. O. Pohl, Phys. Rev. B 59, 11767 (1999).

${ }^{32}$ A. B. Hutchinson, P. A. Truitt, K. C. Schwab, L. Sekaric, J. M. Parpia, H. G. Craighead, and J. E. Butler, Appl. Phys. Lett. 84, 972 (2004).

${ }^{33}$ X. L. Feng, C. A. Zorman, M. Mehregany, and M. L. Roukes, Tech. Digest, Solid-State Sensors, Actuators and Microsystems Workshop
(Hilton Head 2006), 342-346, South Carolina, June 4-8 (2006); e-print arXiv:hep-th/0606711.

${ }^{34}$ B. E. White, Jr. and R. O. Pohl, Phys. Rev. Lett. 75, 4437 (1995).

${ }^{35}$ A. Olkhovets, S. Evoy, D. W. Carr, J. M. Parpia, and H. G. Craighead, J. Vac. Sci. Technol. B: Microelectron. Nanometer Struct. 18, 3549 (2000).

${ }^{36}$ F. Hoehne, Y. A. Pashkin, O. Astafiev, L. Faoro, L. B. Ioffe, Y. Nakamura, and J. S. Tsai, Phys. Rev. B 81, 184112 (2010).

${ }^{37}$ A. Venkatesan, K. J. Lulla, M. J. Patton, A. D. Armour, C. J. Mellor, and J. R. Owers-Bradley, Phys. Rev. B 81, 073410 (2010).

${ }^{38}$ S. Prabhakar and S. Vengallatore, J. Micromech. Microengineering 17, 532 (2007).

${ }^{39}$ J. L. Arlett, E. B. Myers, and M. L. Roukes, Nat. Nanotechnol. 6, 203 (2011).

${ }^{40}$ Z. Darázs, Z. Kurucz, O. Kálmán, T. Kiss, J. Fortágh, and P. Domokos, Phys. Rev. Lett. 112, 133603 (2014).

${ }^{41}$ E. Collin, J. Kofler, S. Lakhloufi, S. Pairis, Y. M. Bunkov, and H. Godfrin, J. Appl. Phys. 107, 114905 (2010). 